\title{
Whether feeling or seeing is more accurate depends on tracking direction within the perception-action cycle
}

\author{
Kaat Alaerts*, Oron Levin, Stephan P. Swinnen \\ Motor Control Laboratory, Division of Movement Control and Neuroplasticity, Department of Biomedical Kinesiology, \\ Group Biomedical Sciences, Katholieke Universtiteit Leuven, Tervuursevest 101, B-3001 Heverlee, Belgium \\ Received 24 October 2006; received in revised form 19 December 2006; accepted 21 December 2006 \\ Available online 7 January 2007
}

\begin{abstract}
Coordination in biological systems requires a continuous updating of the perception-action cycle. How the different sources of sensory information contribute uniquely to performance is still debated. Here, we directly compared the role of vision and kinesthesis by means of a tracking task in which the left wrist mimicked the passive motions imposed on the right wrist with a torque motor. The passive movements were perceived visually (alien hand) or kinesthetically (own hand), or a combination thereof (own hand, feel and see). Tracking occurred according to the same (isodirectional) or opposite (mirror-image) directions. Findings revealed that visual tracking was performed most successfully in the isodirectional and kinesthetic tracking in the mirror-image mode. Tracking was most successful when both sources of sensory information were present. These results suggest that vision and proprioception obey direction-dependent constraints that are consistent with extrinsic and intrinsic reference frames within which the perception-action cycle resides. Thus, each sensory modality contributes uniquely as a function of the spatial requirements of the tracking task, rather than one being superior over the other.
\end{abstract}

(C) 2007 Elsevier B.V. All rights reserved.

Keywords: Sensory afferents; Coordination principles; Tracking; Vision; Proprioception; Sensory integration; Interlimb coordination

\section{Introduction}

In biological systems, a continuous updating of the perception-action cycle is required to generate purposeful context-dependent actions. Among the different sensory modalities, vision and proprioception are most critical for planning and guiding movements. Both sources of sensory information are usually integrated to generate a single estimate of limb position in space. How these sources of sensory information relate to each other and what their unique contribution is, remains a matter of debate. The traditional view has been that vision dominates proprioception but alternative evidence exists [33].

More recently, the role of sensory information has also been debated intensively within the context of movement coordination $[18,30,35]$. Paradigms addressing coordination dynamics between two effectors have been most prominent. This has resulted in the identification of stable coordina-

\footnotetext{
* Corresponding author. Tel.: +32163290 66; fax: +32 16329197.

E-mail address: kaat.alaerts@ faber.kuleuven.be (K. Alaerts).
}

tion states, generally referring to the in-phase and anti-phase modes. Intermediate modes are less stable and the differences in stability become amplified with increases in movement frequency [12]. For example, in bimanual coordination, the simultaneous timing of homologous muscle activation (inphase, mirror-image mode with respect to the midline of the body) results in more stable coordination than alternated activation (anti-phase, parallel mode) of these muscle groups [9,15,29,32]. Besides the stabilizing effect of muscle grouping, spatial constraints (isodirectionality) have also been reported to contribute to the stabilisation of these coordination dynamics $[18,22,32]$. More specifically, bimanual isodirectional movements involving homologous muscle groups are more stable than non-isodirectional movements involving non-homologous muscle groups, with the remaining conditions (when only one of both principles applies) positioned in between [29,30]. In general, the muscle grouping constraint prevails over the isodirectional constraint during bimanual coordination.

The prominence of isodirectional coupling has also been documented during coordination of an effector with a visual target or during between-person coordination $[31,32]$. Tracking tasks 
requiring movement of the active limb in the same direction in extrinsic space as the visual stimulus are performed more accurately than tasks involving movement in opposite directions $[8,20,23,32,37]$. This is consistent with a more generic principle in visual perception whereby isodirectional stimuli are more preferably grouped into a perceptual unit or 'gestalt' than non-isodirectional stimuli $[4,14,36]$. However, when applied to bimanual coordination, perceptual grouping of visual feedback stimuli does not always result in a stabilisation of coordination [7], because this is also mediated by the degree of congruency between the performed pattern and the perceived visual feedback [24,25].

The aforementioned observations have led to speculations about the mediating role of afferents and efferents in these general coordination principles. Interestingly, Bingham et al. had participants judge the degree of stability in two visually presented oscillating dots and found that the judgments of phase variability varied according to an asymmetric U-shaped function of mean relative phase, i.e., a mean relative phase of $180^{\circ}$ was judged to be intrinsically more variable than a mean relative phase of $0^{\circ}$ [3]. The observed similarity between the aforementioned movement coordination and perception results led them to conclude that the movement coordination principles were partly mediated by perceptual processes. Others have expressed a stronger position in this debate, suggesting that the movement coordination phenomena are solely due to perception [18]. However, what has remained largely unresolved so far is whether and how the visual and kinesthetic afferents contribute uniquely to coordinated behaviour in general and to the preference for distinct directional relationships between the moving limbs in particular.

In the past, various paradigms have addressed the role of sensory information by assessing changes in motor performance as a result of removing or distorting the role of vision (prism glasses) and/or proprioception (tendon vibration), or by studying specific patient groups. Here, we used a tracking task in which the role of visual and proprioceptive information as well as their combination was directly compared within a perception-action setup. The task required the left wrist to match, as accurately as possible, the cyclical passive motion imposed on the right wrist by means of a torque motor. The passive movement consisted of a superposition of sine waves and was perceived visually (alien hand) or kinesthetically (own hand), or a combination thereof (own hand, feel and see). Participants tracked the imposed wrist motion according to the same (isodirectional) or opposite (mirror-image) directions. Finally, tracking performance was assessed at three different cycling frequencies $(0.5,1.0$, and $1.5 \mathrm{~Hz}$ ) to determine how the different sensory modalities coped with increased time pressure.

It was hypothesized that availability of both sensory modalities resulted in superior perception of limb motion as compared to each modality separately as a result of inter-sensory integration. With respect to tracking direction, visual tracking was hypothesized to be superior in the isodirectional mode and proprioceptive tracking in the non-isodirectional or mirror-image mode. Finally, increases in speed were predicted to result in a more prominent performance deterioration in the separate as compared to the combined sensory modalities. Even though the present tracking task differs substantially from a typical bimanual coordination task, the results will be interpreted in view of the currently known coordination principles.

\section{Materials and methods}

\subsection{Subjects}

Participants were 10 male and 8 female volunteers (aged 19-27 years) without any known neuromuscular disorders. All participants were right-handed, as assessed with the Edinburgh Handedness Questionnaire [19] and were naive about the purpose of the experiment. Informed consent was obtained before the experiment, and the experimental procedure was approved by the local Ethics Committee for Biomedical Research at the Katholieke Universiteit Leuven.

\subsection{Task}

A purpose-built apparatus (see Fig. 1) was used to impose flexion-extension movements to the right wrist. The apparatus consisted of two separate units (left and right), both fitted with a forearm rest to support it in a natural position, and a hand piece for insertion of the hand palm. Motion of the right wrist joint was induced by means of an AC servo motor (AMK DV764, Goedhard PMC, Helmond, NL) that was mounted underneath the right unit and coupled to the rotating shaft of the manipulandum via a 1:10 redactor (Alpha Gearbox, Type LP120). The motor generated a continuous but irregular sinusoidal motion of a programmable amplitude, frequency and duration, allowing rotation of the wrist from $-30^{\circ}$ (flexion) to $+30^{\circ}$ (extension), relative to a $0^{\circ}$ position (whereby the forearm and the palmar surface of the hand were aligned). The movement consisted of a superposition of sine waves with varying amplitude and frequency and varied form trial to trial to prevent prediction and anticipation (see Fig. 2).

The left hand piece was constructed similarly but enabled free rotation of the wrist. Shaft encoders were connected to the rotating axis (accuracy $=0.088^{\circ}$ ) to record angular displacement of the left and right wrist. Data were sampled at $1000 \mathrm{~Hz}$ (Signal software, Version 2.02; Cambridge Electronic Design,

(A)

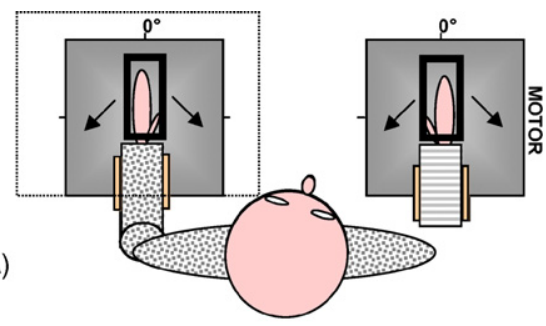

(B)

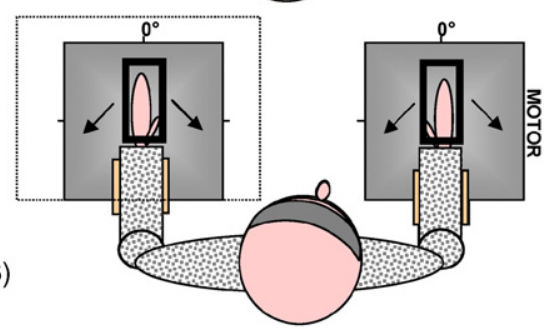

(C)

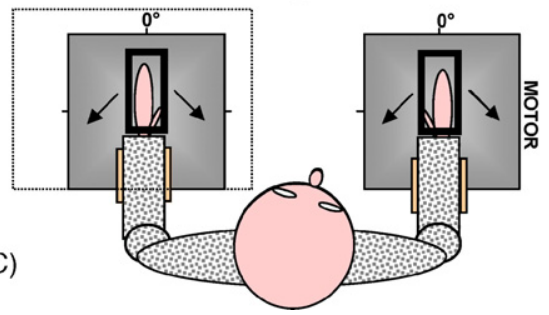

Fig. 1. Schematic view of the experimental conditions: (A) visual tracking, (B) kinesthetic tracking, and $(\mathrm{C})$ visuo-kinesthetic tracking. 


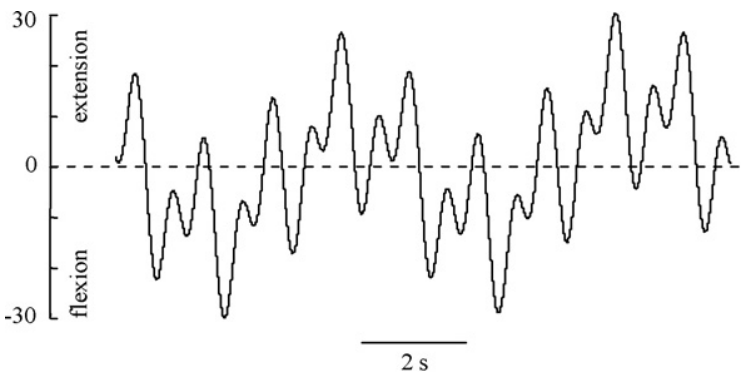

Fig. 2. Example trace of the imposed passive movement on the right limb.

Cambridge, UK) and low-pass filtered (second-order Butterworth with cut-off frequency at $8 \mathrm{~Hz}$, with zero-lag). The angular displacement signals of the two hand pieces were stored for further analysis.

\subsection{Procedure}

Subjects were seated in front of the apparatus with their shoulders positioned in slight abduction $\left(10-20^{\circ}\right)$, elbows at $90^{\circ}$ and forearm supported in a neutral pro-supination position. Participants were instructed to match the movement of the motor-driven right hand with their left hand as accurately as possible, taking into account amplitude, velocity and direction.

Tracking was performed according to three sensory conditions (i.e., visual, kinesthetic and visuo-kinesthetic) and two directional requirements (i.e., isodirectional and non-isodirectional motion). The isodirectional mode (ISO) referred to matching of the right hand with the left hand by means of motion in the same direction in extrinsic space (i.e., a non mirror-image mode), whereas the non-isodirectional mode (N-ISO) required motion of the two hands in opposite directions in extrinsic space (i.e., a mirror-image mode, relative to the midline of the body).

The three sensory conditions referred to the sensory modality afforded by the target (right) hand, as illustrated in Fig. 1. In the visual condition, subjects were instructed to match with their left hand the visually perceived motion of the right hand of a third person that was inserted into the hand piece (visual tracking alien hand). The third person (female, aged 23) inserted her hand into the hand piece as if it were the subject's own hand. The third person was positioned lower than the performer to avoid distraction. Moreover, the participants were instructed not to pay attention to the presence of the third person. In the proprioceptive condition, the subject was blindfolded and could only 'sense' the movement of his own right wrist imposed by the torque motor (kinesthetic tracking own hand). In the third condition, the subject observed and sensed the imposed movement of his/her right wrist (visuo-kinesthetic tracking own hand). Sensory feedback of the actively moved left hand was the same for all tracking tasks, i.e., vision was not allowed such that proprioceptive information prevailed. Accordingly, our notation of the three aforementioned tracking conditions is primarily focused upon the sensory modality(ies) available with respect to right hand movement. Please note that it was not possible to map all right-hand conditions onto the left hand to obtain a more balanced experimental design. For example, focal vision of both hands simultaneously was not possible because of the large betweenhand distance. Therefore, we restricted the left hand conditions to the kinesthetic sensory modality only.

Each tracking task was performed at three different speeds: $0.5 \mathrm{~Hz}$ (slow), $1 \mathrm{~Hz}$ (medium) and $1.5 \mathrm{~Hz}$ (fast). In total, 18 task conditions were presented (3 sensory conditions $\times 3$ speeds $\times 2$ directions). At initiation of each of the 18 experimental conditions, a practice trial was performed, followed by the recording of four test trials. This resulted in a grand total of 72 recorded trials (3 sensory conditions $\times 3$ speeds $\times 2$ directions $\times 4$ trials). A short pause $(5 \mathrm{~min})$ was inserted after 48 trials. To prevent anticipation of the imposed right wrist motion, three different sets of continuous, quasi-random, sinusoidal displacement series (duration of $30 \mathrm{~s}$ ) were used randomly across the 72 recorded trials. The quasi-random character of each of the displacement series was ensured by varying the amplitude continuously between $\pm 30^{\circ}$ (see Fig. 2). The order of presentation of the test trials was also randomised for each subject.

During tracking, participants were instructed to maintain their driven right hand 'as relaxed as possible'. Although electromyographic (EMG) recordings of muscle activity of the passively moved hand were not monitored in the present study, previous pilot work revealed that there was no overt muscle activity of the passively moved hand during kinesthetic tracking (unpublished data).

\subsection{Analysis of tracking performance}

\subsubsection{Relative phase}

The accuracy and consistency of tracking performance was assessed by means of relative phase measurements. The relative phasing between joint angle pairs was obtained from the instantaneous phase of each signal, derived from the Hilbert transform $[5,6,10]$. Relative phase was defined as the subtraction of the phase angle of the left (actively moved) from the right (passively moved) wrist according to the following formula [16]:

$\Phi=\theta_{\mathrm{RW}}-\theta_{\mathrm{LW}}=\tan ^{-1}\left[\frac{\left(\mathrm{d} X_{\mathrm{RW}} / \mathrm{d} t\right)}{X_{\mathrm{RW}}}\right]-\tan ^{-1}\left[\frac{\left(\mathrm{d} X_{\mathrm{LW}} / \mathrm{d} t\right)}{X_{\mathrm{LW}}}\right]$

where $\theta_{\mathrm{RW}}$ refers to the phase of the right wrist movement at each sample, $X_{\mathrm{RW}}$ is the position of the right wrist after rescaling to the interval $[-1,1]$ for each cycle of oscillation, and $\mathrm{d} X_{\mathrm{RW}} / \mathrm{d} t$ is the normalized instantaneous velocity. Subsequently, measures of tracking accuracy and consistency were determined. The mean absolute error of relative phase (AE $\Phi$ ) reflected the deviation from the target relative phase $\left(0^{\circ}\right.$ for ISO tracking and $180^{\circ}$ for $\mathrm{N}$-ISO tracking) (tracking accuracy). The standard deviation of relative phase (SD $\Phi$ ) referred to the spread of relative phase measures around the mean (tracking consistency).

\subsubsection{Amplitude deviation}

Values of amplitude deviation (AMPDEV) between the perceived (motordriven) motion and the corresponding active (left hand) motion were derived from the continuous displacement series of each hand (sampling rate $=100 \mathrm{~Hz}$ ) and refer to the accuracy of amplitude tracking. The AMPDEV scores were calculated according to the following formula: AMPDEV $=$ mean $\left(\left|X_{\mathrm{RW}}\right|-\left|X_{\mathrm{LW}}\right|\right)$, whereby $X_{\mathrm{RW}}$ is the angular position of the right wrist and $X_{\mathrm{LW}}$ is the angular position of the left wrist.

\subsection{Statistical analyses}

The AE $\Phi$, SD $\Phi$ and AMPDEV scores were determined for each trial and subsequently averaged across the four test trials of each condition. Three-way ( 3 sensory condition $\times 3$ speed $\times 2$ direction) analyses of variance for repeated measures (ANOVA) were performed using Statistica software (version 6.0). Significant results of interest were examined post-hoc with planned comparisons (PC) (equivalent to the $t$-test for independent samples) with Bonferroni correction. Other significant results were explored with Tukey HSD post-hoc tests. The $\alpha$-level of significance was set to $\alpha=0.05$.

\section{Results}

\subsection{Absolute error of relative phase (AEФ)}

The $3 \times 3 \times 2$ ANOVA of AE $\Phi$ revealed a main effect of sensory condition $[F(2,34)=6.87, p=0.003]$, indicating that AE $\Phi$ was significantly lower in the visuo-kinesthetic tracking condition $(\mathrm{V}+\mathrm{KT})\left(M=39.35^{\circ}, \mathrm{SE}=1.64\right)$ as compared to the visual (VT) $\left(M=45.27^{\circ}, \mathrm{SE}=1.70\right)$ or kinesthetic tracking condition (KT) $\left(M=44.31^{\circ}, \mathrm{SE}=1.55\right)$ [planned comparisons with Bonferroni correction: $F(1,17)=19.87, p=0.00$ and $F(1$, $17)=6.61, p=0.04]$. However, a highly significant sensory condition $\times$ direction interaction $[F(2,34)=10.455, p=0.00]$ indicated that this effect varied as a function of the directional requirement (i.e., ISO versus N-ISO) (see Fig. 3A). Post-hoc analysis (planned comparison with Bonferroni correction) revealed that $\mathrm{AE} \Phi$ was significantly lower for $\mathrm{V}+\mathrm{KT}$ $\left(M=38.24^{\circ}, \mathrm{SE}=2.02\right)$ than for $\mathrm{VT}\left(M=47.57^{\circ}, \mathrm{SE}=1.99\right)$ in 

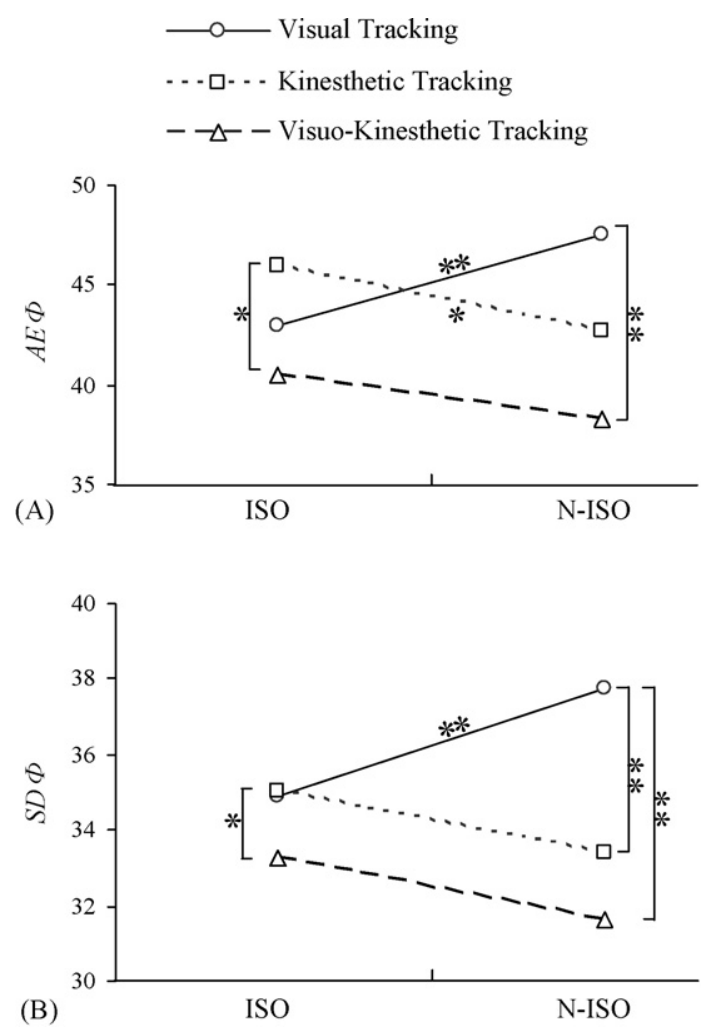

Fig. 3. The sensory condition $\times$ direction interaction for AE $\Phi(\mathrm{A})$ and $\mathrm{SD} \Phi(\mathrm{B})$ $(n=18)$. ISO isodirectional, N-ISO non-isodirectional; ${ }^{* *} p<0.01$.

the N-ISO mode $[F(1,17)=25.48, p=0.00]$, whereas AE $\Phi$ was lower for $\mathrm{V}+\mathrm{KT}\left(M=40.54^{\circ}, \mathrm{SE}=1.51\right)$ than for $\mathrm{KT}$ $\left(M=45.94^{\circ}, \mathrm{SE}=1.39\right)$ in the ISO mode $[F(1,17)=15.48$, $p=0.0032]$.

The aforementioned interaction also allowed us to look into differential direction effects within each sensory condition (see Fig. 3A). Visual tracking (VT) was performed with significantly smaller absolute error scores in the ISO $\left(M=42.97^{\circ}, \mathrm{SE}=1.52\right)$ as compared to the N-ISO mode $\left(M=47.57^{\circ}, \mathrm{SE}=1.99\right)[F(1$, $17)=22.167, p=0.0012]$. Conversely, during kinesthetic tracking (KT), AE $\Phi$ scores were lower during N-ISO $\left(M=42.68^{\circ}\right.$, $\mathrm{SE}=1.98)$ than during ISO tracking $\left(M=45.94^{\circ}, \mathrm{SE}=1.39\right)$ $[F(1,17)=5.129, p=0.037]$. No difference in AE $\Phi$ between tracking in the ISO $\left(M=40.54^{\circ}, \mathrm{SE}=1.51\right)$ and N-ISO mode $\left(M=38.24^{\circ}, \mathrm{SE}=2.02\right)$ was observed during $\mathrm{V}+\mathrm{KT}[F(1$, 17) $=2.443, p>.1]$.

The main effect of speed was highly significant, indicating that tracking accuracy deteriorated with increasing speed $[F(2$, $34)=397.82, p=0.00]$. In addition, the speed $\times$ sensory condition interaction was also significant $[F(4,68)=3.10, p=0.022]$. Post-hoc tests (Tukey HSD) revealed that the aforementioned sensory condition effect was slightly more pronounced at higher speeds. The three-factor interaction was not significant $[F(4$, 68) $=1.70, p>.1]$.

\subsection{Standard deviation of relative phase (SDФ)}

Overall, the SD $\Phi$ scores showed similar tendencies as those found for AE $\Phi$ (see Fig. 3B). The main effect of sensory con- dition was significant $[F(2,34)=21.45, p=0.00]$, as well as the sensory condition $\times$ direction interaction $[F(2,34)=10.07$, $p=0.00]$. Post-hoc analysis revealed that $\mathrm{V}+\mathrm{KT}\left(M=31.61^{\circ}\right.$, $\mathrm{SE}=0.88)$ ] showed significantly less relative phase variability than VT $\left[M=37.79^{\circ}, \mathrm{SE}=0.75\right)$ in the N-ISO mode, whereas $\mathrm{V}+\mathrm{KT}\left[M=33.24^{\circ}, \mathrm{SE}=0.53\right.$ ) showed less variability than $\mathrm{KT}$ $\left(M=35.02^{\circ}, \mathrm{SE}=0.55\right)$ in the ISO mode. In addition, post-hoc analysis indicated that KT exhibited lower variability of relative phase than VT in the N-ISO mode $[F(1,17)=24.17, p=0.00]$, whereas tracking in the ISO mode was performed equally consistent in the KT and VT condition $[F(1,17)=0.02, p=0.88]$.

The sensory condition $\times$ direction interaction revealed also that for VT, SD $\Phi$ was significantly smaller in the ISO mode $\left(M=34.91^{\circ}, \mathrm{SE}=0.66\right)$ than in the N-ISO mode $\left(M=37.79^{\circ}\right.$, $\mathrm{SE}=0.75)[F(1,17)=21.35, p=0.00]$. Marginally significant differences in SD $\Phi$ between tracking in the ISO and N-ISO mode were found for $\mathrm{V}+\mathrm{KT}[F=(1,17)=3.42, p=.082]$ and KT $[F=(1,17)=3.10, p=0.096]$.

The main effect of speed revealed that consistency of all matching tasks severely deteriorated with increasing speed $[F(2$, $34)=250.57, p=0.00]$. None of the remaining main effects or interactions reached significance [all, $p>0.2$ ].

\subsection{Amplitude deviation (AMPDEV)}

The $3 \times 3 \times 2$ ANOVA revealed main effects of sensory condition $[F(2,34)=8.26 ; p=0.012]$, direction $[F(2,34)=53.06$; $p=0.00]$, and speed $[F(2,34)=42.64 ; p=0.00]$. The first two effects resulted from, respectively, larger amplitude overshoot in VT $\left(M=5.01^{\circ}, \mathrm{SE}=0.52\right)$ as compared to $\mathrm{KT}\left(M=2.37^{\circ}\right.$, $\mathrm{SE}=0.57)$ and $\mathrm{V}+\mathrm{KT}\left(M=2.86^{\circ}, \mathrm{SE}=0.50\right)$, and a larger amplitude overshoot in the ISO $\left(M=4.49^{\circ}, \mathrm{SE}=0.41\right)$ as compared to the N-ISO mode $\left(M=2.33^{\circ}, \mathrm{SE}=0.35\right)$. AMPDEV scores were significantly larger during tracking at medium $\left(M=4.73^{\circ}, \mathrm{SE}=0.33\right)$ and fast $\left(M=5.05^{\circ}, \mathrm{SE}=0.58\right)$ as compared to low speeds $\left(M=0.46^{\circ}\right.$, SE 0.48$)$.

In addition, a significant sensory condition $\times$ speed interaction $[F(4,68)=2.97 ; p=0.025]$ was found. Post-hoc (Tukey HSD) exploration of this interaction revealed that the aforementioned sensory condition effect was only significant at the highest speed $(1.5 \mathrm{~Hz})$. The three-factor interaction was not significant $[F(4,86)<1]$.

\section{Discussion}

In the present study, the role of sensory modalities in the context of the perception-action cycle was investigated by means of a tracking paradigm in which participants matched with their left hand the passive movements imposed on their own (feel, or see and feel) or an alien's right hand (see). To our knowledge, this is the first time that the role of these sensory modalities is directly compared in the context of unimanual tracking. Superior tracking performance was observed when vision and kinesthesis were simultaneously available as compared to each sensory modality separately. Furthermore, the superiority of each sensory modality was shown to depend on tracking direction: vision was superior during isodirectional tracking and kinesthesis dur- 
ing non-isodirectional (mirror-image) tracking. Whereas similar principles of sensory processing across modalities are sometimes implicitly assumed [18], the present findings shed light on the unique impact of both sources of sensory information on direction-dependent constraints during the perception-action cycle. Moreover, even though the present tracking task differs from a typical interlimb coordination task, the observations afford a deeper understanding of the general coordination principles, as discussed next.

\subsection{Tracking of movement direction}

Tracking of direction was primarily assessed by the relative phase relationship between the tracked and tracking limb. Our results clearly supported distinctive direction-dependent constraints in visual and kinesthetic tracking. Visual tracking of the alien hand was performed more accurately when the active and passive limb moved in the same direction in extrinsic space (isodirectional) as compared to motion in opposite directions (non-isodirectional), as confirmed by the absolute error and SD scores of relative phase. In contrast, kinesthetic tracking was more accurate under the non-isodirectional (i.e., mirror-symmetry with respect to the midline of the body) than isodirectional mode, as inferred from the absolute error of relative phase.

The superiority of isodirectional tracking under visual information conditions is consistent with previous work in coupling an effector with an external visual stimulus [8,20,23,32,37]. Performance was found to be more optimal when the limb and visual signal were moved in the same as compared to different coupling directions. This is reminiscent of a more general principle in visual organization in perceptual gestalt theory, known as the 'common fate law'. Wertheimer suggested that moving stimuli in the same direction and with the same speed are observed as perceptual units [34]. In line with this observation, Johannson found that when four $(2 \times 2)$ stimuli moved together up- and downwards, the stimuli moving into the same direction were perceptually grouped as a whole whereas the stimuli moving in opposite directions were not [13]. Similarly, when watching a group of moving stimuli, the sub-clusters with a common movement direction were more easily recognized and detected than those that were not $[1,2,17]$. Accordingly, Bingham et al. showed that perception of visual stimuli moving in the same direction were judged as being more stable than those in different directions [3]. Thus, the general evidence suggests that visual stimuli moving in the same direction are perceptually more salient than other motion structures and this principle extends to perceptionaction coupling conditions in which a visual stimulus is tracked with a limb. Similarly, in ergonomics, 'direct drive' (isodirectionality) between movement of the control and the display is a very prominent principle in control-display relations to promote optimal performance [26].

In spite of the prominence of the isodirectional principle in visual tracking, kinesthetic tracking was more successful during the mirror-image than during the isodirectional mode. Using a kinesthetic tracking task in which one limb was synchronized with passive motion of the contralateral limb, Stinear found that non-isodirectional tracking was performed with greater stability and accuracy than isodirectional tracking [27]. However, this effect was only marginally confirmed in a later study [21]. The observed preference for mirror-image tracking is consistent with findings in bimanual coordination whereby a strong tendency toward mirror-symmetric motion with respect to the midline of the body has been observed [15,28,29,32]. As the latter coordination mode requires simultaneous engagement of homologous muscle groups in reference to the body midline, this mirror-symmetry tendency or 'egocentric constraint' may result from crossed facilitation of motor pathways between homologous muscles [11]. The allocentric constraint, on the other hand, refers to a preference for isodirectional coupling $[7,28]$. To summarize, our findings lend support to the hypothesis that direction is primarily encoded relative to an intrinsic, egocentric reference frame when proprioceptive afferent information prevails. Conversely, vision affords movement encoding preferably relative to an extrinsic or allocentric reference frame.

Comparing the different sensory modalities, no absolute supremacy of one sensory modality over the other was evident. Rather, a selective direction-dependent preference was observed in which kinesthetic tracking was more successful during the non-isodirectional mode and visual tracking during the isodirectional mode. When comparing combined visuo-kinesthetic tracking with tracking under either modality alone, a significant improvement of tracking performance was observed and direction-dependent differences disappeared: adding vision to isolated kinesthetic tracking improved tracking in the isodirectional mode significantly, whereas the addition of kinesthesis during visual tracking improved performance under non-isodirectional conditions. Thus, kinesthetic and visual information selectively improved mirror-symmetrical and isodirectional tracking under dual-modality conditions, respectively.

\subsection{Amplitude estimation}

Overall, a general overshoot in amplitude was observed across all three tracking conditions. This was substantially more pronounced for visual as compared to both kinesthetic and visuo-kinesthetic tracking. Thus, amplitude estimation was more successful when proprioceptive afferent information was available. During kinesthetic tracking, the observed amplitude overshoot may have resulted from overestimation of dominant limb afferent sources, as tracking required matching of the motion of the passively driven right (dominant) hand with the non-dominant left hand. The converse arrangement was found to induce amplitude underestimation [27]. Apart from that, our data also indicated a more pronounced amplitude overshoot during isodirectional as compared to non-isodirectional tracking, suggesting a facilitation of amplitude generation under the former conditions. Finally, amplitude overestimation was also more pronounced at increasing speeds, reflecting a general deterioration in estimation of limb position under increased temporal pressure.

In summary, the present observations shed light on the direction-dependent constraints that became evident during perception-action coupling in the context of tracking. Kines- 
thetic tracking was more successful during the mirror-image than isodirectional mode, whereas the converse observation was made during visual tracking. The combination of both sources of sensory information resulted in superior tracking performance in which the direction-specific limitations of each modality were overcome. These direction-dependent sensory effects are reminiscent of two highly prevalent modes of coordination between effectors, namely the allocentric constraint (or preference to move in the same direction in extrinsic space), and the egocentric constraint (or the preference to move both limbs towards and away from the body midline, activating the homologous muscle groups simultaneously) [28]. Because the critical feature of the present tracking task was the perception of (alien or own) motion of the dominant limb and matching with the contralateral limb, it is reasonable to assume that the aforementioned interlimb coordination principles are partly mediated by basic sensory processes that exhibit a direction-dependent preference. The more general message emerging from this observation is that a better understanding of the principles underlying interlimb coordination in complex biological systems is dependent upon a more detailed investigation of the principles underlying different sources of sensory information.

\section{Acknowledgements}

Support for this study was provided through a grant from the Research Council of K.U. Leuven, Belgium (Contract OT/03/61) and the Flanders Fund for Scientific Research (Projects G.0460.04 \& G.0292.05).

\section{References}

[1] Ahlstrom U. Perceptual unit formation in simple motion patterns. Scand J Psychol 1995;36(4):343-54.

[2] Ahlstrom U, Borjesson E. Segregation of motion structure from random visual noise. Perception 1996;25(3):279-91.

[3] Bingham GP, Schmidt RC, Zaal FTJM. Visual perception of the relative phasing of human limb movements. Percept Psychophys 1999;61(2):246-58.

[4] Bingham GP, Zaal FT, Shull JA, Collins DR. The effect of frequency on the visual perception of relative phase and phase variability of two oscillating objects. Exp Brain Res 2001;136(4):543-52.

[5] Boashash B. Estimating and interpreting the instantaneous frequency of A signal. 2. Algorithms and applications. Proc IEEE 1992;80(4):540-68.

[6] Boashash B. Estimating and interpreting the instantaneous frequency of A signal. 1. Fundamentals. Proc IEEE 1992;80(4):520-38.

[7] Bogaerts H, Buekers MJ, Zaal FT, Swinnen SP. When visuo-motor incongruence aids motor performance: the effect of perceiving motion structures during transformed visual feedback on bimanual coordination. Behav Brain Res 2003;138(1):45-57.

[8] Buekers MJ, Bogaerts HP, Swinnen SP, Helsen WF. The synchronization of human arm movements to external events. Neurosci Let 2000;290(3):181-4.

[9] Carson RG. The dynamics of isometric bimanual coordination. experimental. Brain Res 1995;105(3):465-76.

[10] Carson RG, Smethurst CJ, Forner M, Meichenbaum DP, Mackey DC. Role of peripheral afference during acquisition of a complex coordination task. Exp Brain Res 2002;144(4):496-505.

[11] Carson RG, Welsh TN, Pamblanco-Valero MA. Visual feedback alters the variations in corticospinal excitability that arise from rhythmic movements of the opposite limb. Exp Brain Res 2005;161(3):325-34.
[12] Haken H, Kelso JAS, Bunz H. A theoretical-model of phase-transitions in human hand movements. Biol Cybern 1985;51(5):347-56.

[13] Johansson G. Configurations in event perception. Uppsala: Almqvist and Wiksell; 1950.

[14] Johansson G. Visual motion perception. Sci Am 1975;232(6):76.

[15] Kelso JAS. Phase-transitions and critical-behavior in human bimanual coordination. Am J Physiol 1984;246(6):1000-4.

[16] Kelso JAS, Scholz JP, Schoner G. Nonequilibrium phase-transitions in coordinated biological motion-critical fluctuations. Phys Lett A 1986;118(6):279-84.

[17] Mather G, Moulden B. Thresholds for movement direction: two directions are less detectable than one. Quart J Exp Psychol A 1983;35:513-8.

[18] Mechsner F, Kerzel D, Knoblich G, Prinz W. Perceptual basis of bimanual coordination. Nature 2001;414(6859):69-73.

[19] Oldfield RC. The assessment and analysis of handedness: the Edinburgh inventory. Neuropsychologia 1971;9(1):97-113.

[20] Peper CLE, Beek PJ. Are frequency-induced transitions in rhythmic coordination mediated by a drop in amplitude? Biol Cybern 1998;79(4):291300.

[21] Ridderikhoff A, Peper CL, Beek PJ. Unraveling interlimb interactions underlying bimanual coordination. J Neurophysiol 2005;94(5):3112-25.

[22] Riek S, Carson RG, Byblow WD. Spatial and muscular dependencies in bimanual coordination. J Human Movement Stud 1992;23:251-65.

[23] Roerdink M, Peper CE, Beek PJ. Effects of correct and transformed visual feedback on rhythmic visuo-motor tracking: tracking performance and visual search behavior. Hum Mov Sci 2005;24(3):379-402.

[24] Salesse R, Temprado JJ. The effect of visuo-motor transformations on handfoot coordination: evidence in favor of the incongruency hypothesis. Acta Psychol 2005;119(2):143-57.

[25] Salter JE, Wishart LR, Lee TD, Simon D. Perceptual and motor contributions to bimanual coordination. Neurosci Lett 2004;363(2):102-7.

[26] Sanders MS, McCormick EJ. Human factors in ergonomics and design. 7th ed. New York: McGraw Hill; 1993.

[27] Stinear JW, Byblow WD. Phase transitions and postural deviations during bimanual kinesthetic tracking. Exp Brain Res 2001;137(3/4):467-77.

[28] Swinnen SP, Jardin K, Meulenbroek R, Dounskaia N, HofkensVanDenBrandt M. Egocentric and allocentric constraints in the expression of patterns of interlimb coordination. J Cognitive Neurosci 1997;9(3): 348-77.

[29] Swinnen SP, Jardin K, Verschueren S, Meulenbroek R, Franz L, Dounskaia $\mathrm{N}$, et al. Exploring interlimb constraints during bimanual graphic performance: effects of muscle grouping and direction. Behav Brain Res 1998;90(1):79-87.

[30] Swinnen SP, Puttemans V, Vangheluwe S, Wenderoth N, Levin O, Dounskaia N. Directional interference during bimanual coordination: is interlimb coupling mediated by afferent or efferent processes. Behav Brain Res 2003;139(1-2):177-95

[31] Temprado JJ, Laurent M. Attentional load associated with performing and stabilizing a between-persons coordination of rhythmic limb movements. Acta Psychol 2004;115(1):1-16.

[32] Temprado JJ, Swinnen SP, Carson RG, Tourment A, Laurent M. Interaction of directional, neuromuscular and egocentric constraints on the stability of preferred bimanual coordination patterns. Hum Mov Sci 2003;22(3):339-63.

[33] Welch RB, Warren DH. Intersensory interactions. In: Boff KR, Kaufman L, Thomas JP, editors. Handbook of perception and human performance. 1986. p. 25.1-36.

[34] Wertheimer M. Untersuchungen zur Lehre von der Gestalt. Psychol Forsch 1923;4:301-50.

[35] Wilson AD, Bingham GP, Craig JC. Proprioceptive perception of phase variability. J Exp Psychol Hum Percept Perform 2003;29(6):117990.

[36] Wilson AD, Collins DR, Bingham GP. Perceptual coupling in rhythmic movement coordination: stable perception leads to stable action. Exp Brain Res 2005;164(4):517-28.

[37] Wimmers RH, Beek PJ, Vanwieringen PCW. Phase-transitions in rhythmic tracking movements-a case of unilateral coupling. Hum Move Sci 1992;11(1/2):217-26. 